\title{
Automated FAQ Answering with Question-Specific Knowledge Representation for Web Self-Service
}

\author{
Eriks Sneiders \\ Dept. of Computer and Systems Sciences, Stockholm University / KTH, Sweden \\ eriks@dsv.su.se
}

\begin{abstract}
Automated FAQ answering is a valuable complement to web self-service: while the vast majority of site searches fail, our FAQ answering solution for restricted domains answers two thirds of the queries with accuracy over $90 \%$. The paper is shaped as a best practice summary. The reader will find out how the shortcomings of site search are overcome by FAQ answering, what kind of techniques for FAQ retrieval are available, why question-specific knowledge representation is arguably most appropriate for restricted domains, and what peculiarities of running an FAQ answering service for a customer service may be expected.
\end{abstract}

Keywords - Automated question answering, FAQ answering, FAQ retrieval, web self-service.

\section{INTRODUCTION}

$\mathrm{T}$ HE benefits of web self-service are well known: costs per transaction saved 20-30 times [1] [2], availability around the clock, shorter response time. These benefits hold, however, only if the users find what they are looking for. People using self-service expect quick solutions or abandon the site [3].

Automated question answering (QA) is a step beyond search engines where the system not just retrieves documents but rather delivers answers to user questions. Most often QA stands for fact extraction from free text documents in open domain, or ontology and reasoning based systems in restricted domain. Our research subject is a subclass of QA - automated FAQ (Frequently Asked Questions) retrieval in a restricted domain. The system has been in operation for a few years and has brought up a great deal of positive experience. In the next section we discuss shortcomings of site search engines that FAQ retrieval is meant to overcome. Section III shows the reoccurring nature of user queries, while Section IV gives an insight into various techniques for FAQ retrieval. Section $\mathrm{V}$ presents the main points of our own FAQ retrieval technique, its performance, and the core features.

Sneiders, E. (2009) Automated FAQ Answering with Question-Specific Knowledge Representation for Web Self-Service. Proceedings of the 2nd International Conference on Human System Interaction (HSI'09), May 21-23, Catania, Italy, IEEE, pp.298-305
Section VI is titled "Lessons learned". The advantages of automated FAQ answering for web self-service are listed in Section VII. Two final sections present the further research and conclusions.

\section{SEARCh Is NOt ENOUGH}

Although $82 \%$ of visitors use site search, as much as $85 \%$ of searches do not return the results sought [4]. Search technology is continuously developing by adding spelling correction, synonym vocabularies, processing inflectional forms of words, stemming, compound splitting, analysis of document structure and links between documents. Still, site search has shortcomings caused not by bad technology but rather inherent to keyword-based search phenomenon as such.

1. Information not published. The task of a search engine is to find documents that satisfy the query, a search engine pulls information from a document repository. Unfortunately, there is no guarantee that any relevant documents exist. Analysis of user queries in our case study showed that $65 \%$ of the queries considered for inclusion in the FAQ database did not have any corresponding information published. There is no way the most perfect search technology would ever find it.

2. Wrong keywords. Documents and user queries may refer to the same thing using different wording, and there will be no match. Linguistic enhancements such as synonym dictionaries and stemming do help, yet they hit the ceiling when the keywords change their meaning in different contexts.

3. Documents vs. answers. A search engine finds documents written from information provider's point of view. On a website, people most often search in order to find answers to their questions rather than retrieve documents.

4. Undetermined nature of "0 documents found". This is a consequence of the above features from the user perspective. The fact that no documents are found does not mean that the answer is not possible. For example, a user is searching for a product on a company website and finds no relevant web pages. This is not an answer. In order to find the answer, the user calls the customer 


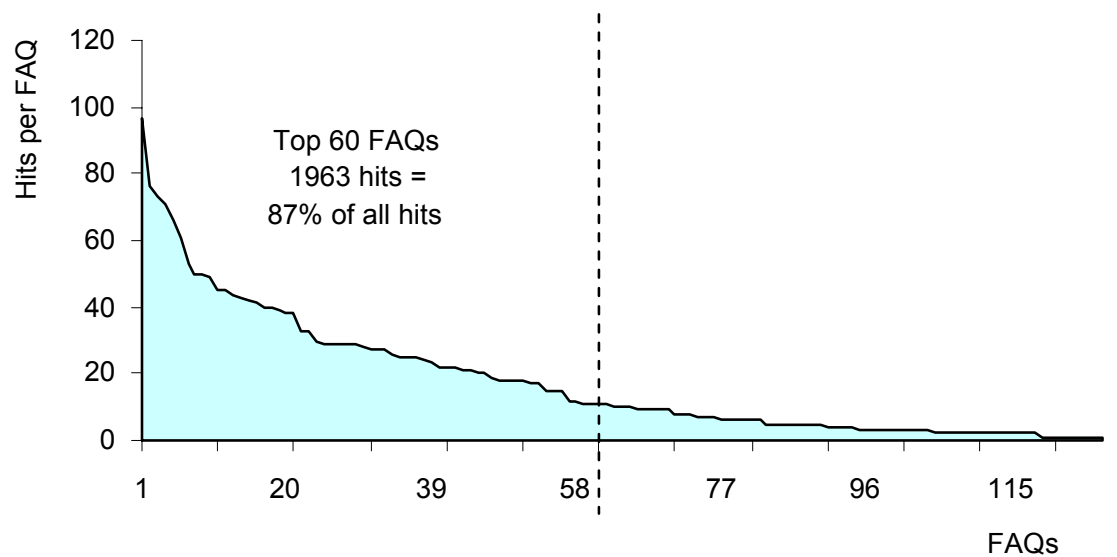

Fig. 1. Distribution of hits per FAQ.

service and finds out that the company does not offer the product. This is a definite answer which could not be delivered by search.

5. Text only. Site search engines retrieve documents in different formats - HTML, plain text, PDF, Microsoft Word, etc. Structured databases and multimedia objects are normally not considered. Search in heterogeneous sources is technically possible [5] and is provided by content management systems such as IBM OmniFind, but not all companies have them or offer outside their closed intranets. Not the least because unrestricted search beyond public web documents is a security threat.

6. Statistical matching methods in a small collection. Search engines use statistical matching methods, they do not interpret user queries or documents. If there is only one relevant document on a website, it is more likely to be buried in search results for common keywords.

7. Short queries. Two studies in restricted domain search report the average query length 2.25 [6] and 2.31 [7] words. It is too little to always retrieve relevant documents.

\section{REOCCURRENCE OF USER QUERIES}

Already when FAQ lists were invented it was clear that people who share the same interests tend to ask the same questions over and over again. Lin [8] notices similarity between reoccurring questions to a QA system and Zipf's curve on word ranks - the curve of question frequencies and Zipf's curve have similar shapes. Furthermore, the whole paradigm of template-based QA relies on reoccurring questions [9].

In order to make the discussion more specific, let us examine the actual user queries recorded in the log files of our FAQ retrieval system. We inspected 1996 consecutive queries; the system answered 1432 queries by matching them to 127 FAQs. In total 2246 hits were generated. A hit is a match between a query and an FAQ. There are more hits than queries because one query can match more than one FAQ and, therefore, generate more than one hit. The distribution of the hits per FAQ is shown in Fig. 1. The top 60 FAQs are responsible for $87 \%$ of all hits. Considering the fact that $72 \%$ of all queries had a hit (1432 of 1996), we can estimate that 60 FAQs matched around $63 \%$ of all queries.

[6] and [10] show frequencies of word pairs from queries in site search, which gives us a hint of popular topics. In another study 25 most popular queries to a US legislation digital library accounted for $17 \%$ of the query flow [7].

Our study shows that the majority of user queries fall into a relatively few subjects that can be successfully covered by FAQs. Furthermore, we can mount an FAQ retrieval system on top of a site search engine and thus increase our users' experience: the search facility starts answering questions, and there is no need to browse FAQ lists.

\section{APPROACHES TO FAQ RETRIEVAL}

Most FAQ retrieval techniques fall into three categories: (i) statistical techniques that learn answering new questions from a large amount of sample data, (ii) Natural Language Processing (NLP) and semantic text analysis, and (iii) template-based techniques where each FAQ has a cluster of templates that mimic expected user questions.

\section{A. Statistical techniques}

Statistical techniques are most useful when we deal with a large number of question-answer pairs created by a third party, for instance those available on the web. The system matches a query to FAQ questions and possibly other parts of the FAQ file/records. Measuring semantic similarity, however, is not trivial because the query and the FAQs may use different wording. For example, "How can I return my merchandise?" and "What do we do in order to get our money back?" carry the same meaning without a single word in common. Therefore the first problem to be solved is bridging lexical gaps, finding which words can be perceived as synonyms in the given context.

[11] tries to resolve lexical gaps by matching the query 
to different fields of an FAQ file - question text, answer text, FAQ page title - with and without stemming, removing stop-words, using n-grams. [12] categorizes previously logged queries using original FAQs as the seed data. The system matches a new query not only to the original FAQs but also to the attached clusters of previous queries, which ensures larger lexical diversity. [13] and [14] are inspired by machine translation techniques. [13] translates FAQs to foreign languages and then back to English, which results in question pairs with the same meaning but sometimes different wording. This way the system learns relationships between words.

After semantic relationships between words are found, the systems calculate similarity between a query and FAQ records using Information Retrieval (IR) methods such as cosine similarity, Okapi BM25, etc.

\section{B. NLP and ontologies}

The NLP approach involves word sense disambiguation and semantic analysis of user queries and FAQ texts, after syntactic, morphological and lexical analysis has been completed. NLP uses manually crafted lexicons and optionally domain ontologies.

The most prominent FAQ retrieval system of this kind is FAQFinder [15] which retrieves question-answer pairs from FAQ files on various topics. The system combines two text similarity measures. One is vector similarity with tf-idf term weights. The other one calculates the distance between words using WordNet. The system attempts to disambiguate nouns and verbs, creates WordNet synonym sets, and then counts the number of hypernym/hyponym links between these synonym sets. The synonym sets and hypernym/hyponym links are general; there is no domain knowledge involved.

[16] uses domain dependent and independent lexicons. Semantic analysis takes the result of lexical analysis of the query in order to detect its semantic category. For each semantic category there is template that computes a representative question for retrieval of the correct answer from the FAQ knowledge base. A concept hierarchy dictionary helps to calculate distance between words. The system learns semantic categories through user feedback.

[17] uses a domain ontology in order to align the concepts in the query with those in the FAQs, as well as to detect FAQs that contain keywords that semantically conflict with the query. Otherwise, probabilistic keyword matching and user feedback are used to calculate similarity between the query and an FAQ entry.

[18] uses a domain ontology in order to calculate semantic distance between words when an FAQ is being categorized or matched to a user query. [19] constructs ontological graphs - connected nodes representing sentences - for the query and FAQs, and compares these graphs.

It should be noted that all FAQ retrieval techniques use basic language processing which may be stemming, spelling checking, morphological normalization, thesaurus, etc.

\section{Template-based techniques}

The most prominent template-based system is START, which makes use of knowledge annotations as "computeranalyzable collections of natural language sentences and phrases that describe the content of various information segments" [20]. An annotation mimics the structure "subject-relationship-object". START matches a user question to the annotation entries on both the word level (using additional lexical information about synonyms, ISA trees, etc.) and the structure level (rules for paraphrased arguments of verbs, nominalization, etc.). When the user question matches an annotation entry, START follows the pointer to the information segment tagged by the annotation and returns it as the answer.

Our system, described in the next section, is another template-based retrieval system.

\section{OUR APPROACH TO FAQ ANSWERING FOR WEB SELF-SERVICE}

Our system encompasses two modules - an FAQ module and a site search engine. The goal of the FAQ module is to capture reoccurring queries, also natural language questions, and present semantically matching answers, while the search engine does its best applying statistical keyword matching to the documents on the website.

The system receives input from two places on the website - "search the site" and "contact us". In "contact us", the users are encouraged to ask question to the system before phoning the customer service. There are three kinds of queries - (i) fully formulated questions, (ii) phrases such as "report the damage", and (iii) single keywords. The FAQ module perceives single keywords as "What can you tell / what should I know about..." type of questions. If the system passes the query to the site search engine, it removes stop-words and too common domain words. If the user has applied "quotation marks", the query is not altered.

The output is following:

- If the FAQ module finds one matching FAQ and is confident about the match, the FAQ and its answer are directly presented to the user. Furthermore, the system offers the user an option to submit a search query, derived form the original user query, to the site search engine.

- If several, say 2-3, FAQs are retrieved or the system is not that confident about the match, the FAQ questions are listed and the user can retrieve their answers one by one. The FAQ list is complemented by a site search result.

- If no matching FAQs are found, the system performs ordinary site search.

The system does not maintain any dialogue the way chatbots do. 


\section{A. Question templates}

The FAQ retrieval technique is described in detail in [21]; minor modifications have been added over the years. A brief description here will help the reader understand the conclusions presented in the following sections.

Unlike other systems that retrieve third party questionanswer pairs, our FAQ module maintains its own database with around 150 FAQs. As we see in Fig. 1, about 60 of them are somewhat often retrieved. Each FAQ has one or several manually crafted question templates (Fig. 2), slightly more than 300 in total. A question template holds linguistic signatures that remotely resemble regular expressions and mimic the language structures of expected user queries.

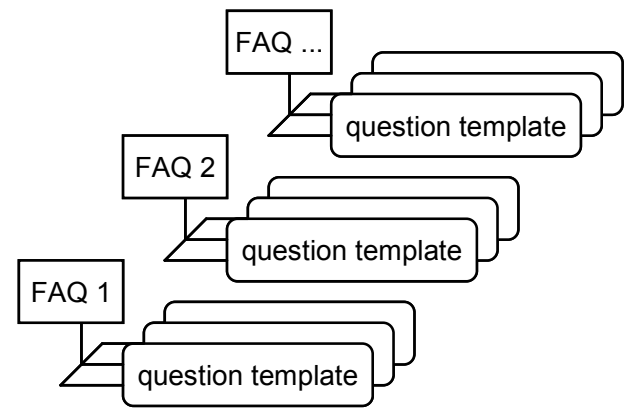

Fig 2. Question templates represent FAQs

A linguistic signature contains synonym sets where a synonym is a word stem, number, or phrase. In a phrase, the order of and distance between synonym sets are defined. A question template has three linguistic signatures that define language structures that (i) must be present, (ii) may be present, and (iii) may not be present in a matching query. By matching these three linguistic signatures to a query the system makes conclusions about the semantic distance between the question template and the query. Several question templates linked to one FAQ cover the lexical diversity of matching queries.

A linguistic signature is the result of syntactic (phrases), morphological (word stemming, compound word splitting), and lexical (synonym sets) analysis of expected user queries. Like all FAQ retrieval techniques do, we also perform linguistic text analysis. Unlike most techniques, we do this analysis before, not after user queries are received. When a query arrives, the system follows the instructions stored in linguistic signatures and matches lexical items to the query.
The system does limited reasoning when it postprocesses retrieved FAQs. It may, for example, disregard simple or low match-confidence FAQs if there are more complex or high match-confidence FAQs retrieved.

\section{B. Performance of FAQ retrieval}

Precision and recall are common IR measures. Recall shows the share of retrieved relevant FAQs among all the relevant FAQs in the database, while precision shows the share of relevant FAQs among all the retrieved FAQs. We measured also rejection, a measure coined by [15]. Rejection shows the share of correctly reported nilanswers when there is no matching FAQ in the database. That is, rejection measures the system's ability not to retrieve garbage if there is no answer in the database.

Precision and recall were measured according to the IR traditions: the retrieved documents - FAQs - were either relevant or not relevant. Such a binary relevance value makes sense because the FAQ database has been optimized for incoming queries over some time and a certain semantic distance between a query and the retrieved FAQs is being deliberately tolerated. We did not judge how well the FAQs were shaped after each query but rather how well the system managed to retrieve relevant and only relevant information choosing from the FAQs that existed in the database at the moment of receiving the query.

We took 317 consecutive queries from the log file and stripped away 112 queries that had no corresponding FAQs in the database. This gave us 205 queries. Precision and recall were measured for each query separately; precision only for the queries with non-zero recall. The aggregated results are shown in Table 1.

The rejection value is the number of correct nil-answers divided by the total number of queries that should have had nil-answers: $97 / 112=0.87$.

From the 16 zero-recall queries, 7 were a re-wording of the previous query from the same IP address within a few seconds. Only 9 zero-recall queries were original information needs.

Our performance measurements show that more than 9 of each 10 queries receive a relevant answer. For comparison, [22] estimates that $70 \%$ is the upper bounds of correctly answered queries in open domain free text QA. Restricted domain QA is supposed to do better than that by applying domain specific knowledge.

In the literature, different performance measures for FAQ-based question answering are used. Furthermore, a system may have several components each evaluated

TABLE 1: QUALITY OF FAQ RETRIEVAL.

\begin{tabular}{cccccc}
\hline Precision & Num queries & Recall & Num queries & Nil-answers & Num queries \\
\hline 1 & 176 & 1 & 186 & Correct & 97 \\
0.50 & 8 & 0.50 & 3 & Garbage & 15 \\
0.67 & 1 & 0 & 16 & & \\
0.33 & 3 & & & & \\
0.25 & 1 & & & & \\
\hline Total queries & 189 & Total queries & 205 & Total queries & 112 \\
Avg precision & 0.96 & Avg recall & 0.91 & Rejection & 0.87 \\
\hline
\end{tabular}


separately, and there may be a number of changing parameters that influence the measurement figures. This makes comparison of different systems tricky. In order to put our own performance measurements into context, we selected some related performance figures that were reasonably easy to interpret.

[11] operates statistical techniques in open domain. Considering the top 20 retrieved FAQs, 36\% of user queries got adequate answers, and $56 \%$ of the queries got relevant information including adequate answers. Considering the top 10 retrieved FAQs, the figures were $29 \%$ and $50 \%$ respectively.

[13] also operates statistical techniques in open domain, in Korean. It has precision 0.78 at recall close to 0 , and precision 0.09 at recall 0.9 . In between, some sample precision/recall values are $0.44 / 0.3$ and $0.21 / 0.6$.

[12] is another Korean system, based on statistical techniques, that operates in a domain of several websites. Its performance was measured by Mean Reciprocal Rank (MRR), which is calculated using the position of the first relevant answer in the list of retrieved FAQs, and so for a number of queries. In a nutshell, MRR value is 1 if all queries get the right answer as the first item in the list of retrieved texts, and 0.5 if the right answer is always the second retrieved text. The best MRR for the answer retrieval component of [12] was 0.7 , given that all other components of the system made no mistakes.

FAQFinder [15] does shallow NLP in open domain. Its question matching component reaches maximum recall 0.68 at 0 rejection. As rejection rises, recall drops. For example, 0.4 rejection results in 0.53 recall, and 0.8 rejection results in 0.25 recall.

[18] is an ontology-based FAQ answering system in restricted domain. It has 0.78 recall at 0.82 rejection.

\section{Question-specific representation of linguistic knowledge}

The main cause of our superior performance figures is granular knowledge representation embodied in question templates. While normally NLP and IR systems work in a vague application-specific knowledge domain, our system works in a number of narrow well-defined FAQ-specific knowledge domains.
Fig. 3 illustrates the difference. On side (b) we have a lexicon and ontology that cover a part of the application knowledge domain. The lexicon has domain dependent and independent parts. The coverage of the knowledge domain must be rather complete if we want to process arbitrary text.

On side (a) we have a number of question templates as pieces of onto-lexical knowledge that patch the application knowledge domain. The coverage of the application knowledge domain is poor and focused only on the most often queried parts; we do not develop knowledge representation that we don't need. Several question templates may share common parts of knowledge representation and thus avoid redundancy.

Question templates encompass the following kinds of knowledge:

- Ontological. Although a question template hardly is an ontology in the sense of Artificial Intelligence, it does define concepts, their attributes, and relationships in the context of the FAQ.

- Lexical. The ontological knowledge is expressed by words and phrases stored in question templates. Context-dependent synonymy and synonymy based on ontological relationships is respected. Slang, abbreviations, localizations, dialects are included.

- Morphological. Words are usually stemmed or the right morphological form is used. Compound words are split.

- Syntactic. Complex syntactic structures are recorded as phrases.

Although question templates all together do not cover much of the application knowledge domain (only the most often queried parts), FAQ-specific linguistic knowledge is pretty well covered because the domain of one template is narrow.

The templates eliminate much of the word sense disambiguation difficulty because synonyms and multiword expressions are defined in narrow context.

Lexical gaps - queries may carry the same meaning while having different wording - are covered by a cluster of templates (see Fig. 2). Such a cluster is analogous query expansion.
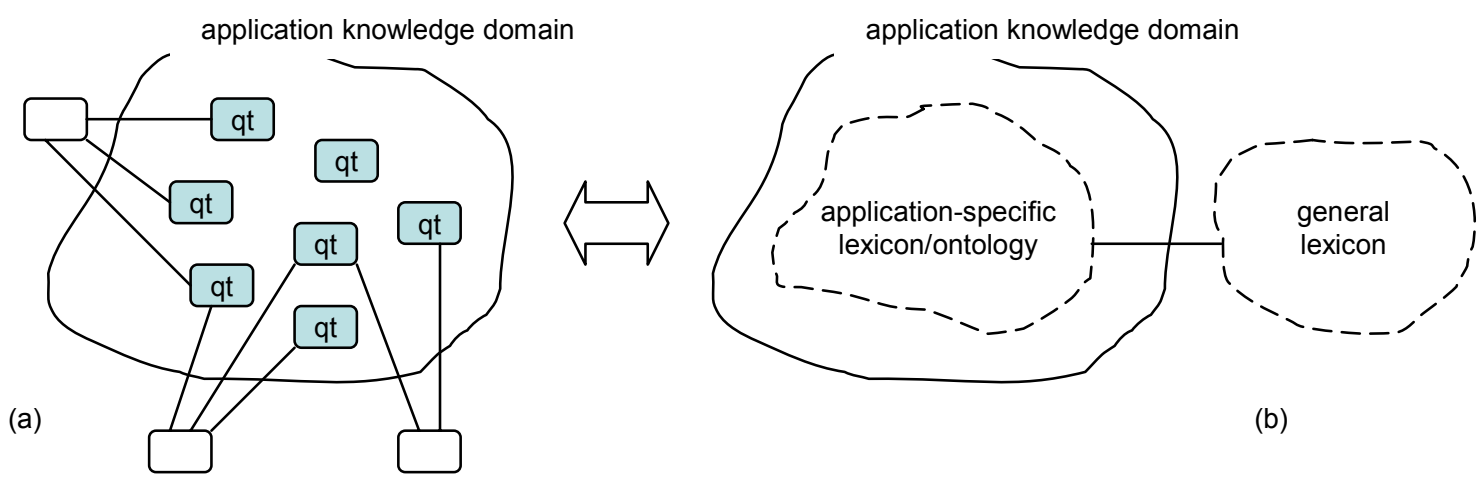

(b)

Fig. 3. FAQ-specific vs. application domain-specific knowledge representation 
Missing words in user queries do not cause problems because the templates assume them in narrow context. For example, "Is it more expensive under 25?" is interpreted as "Is a car insurance more expensive if the driver is under 25 years of age?" in the context of the right FAQ. The templates can deal with short queries such as search phrases and keywords.

Question templates are crafted manually analyzing previous user queries, if such queries are available. In this sense our technique correlates with [12] (see Section IV.A). Nonetheless, the data in a template is not statistical. A template encapsulates human decisions based on (i) statistical information about past queries and (ii) reasoning about the future queries.

Crafting question templates can be compared with Search Engine Optimization where Google-enthusiasts target popular search queries and optimize specific web pages for these queries. For popular queries on major search engines, a web page stands not a chance to reach the top of search results without being heavily optimized. The optimization effort is continuous because search engines regularly modify their ranking criteria. In our case, instead of HTML documents we optimize question templates to capture expected relevant queries and ignore irrelevant ones. The syntax of our linguistic signatures and the matching algorithm do not change.

\section{LESSONS LEARNED}

Our FAQ answering system has been in operation for a few years by now, and we have accumulated some experience to be shared.

\section{A. Starting the FAQ answering service}

As we started building the FAQ database from scratch, the first problem was finding the users' information needs. Site search logs were not available because the search service was outsourced. Therefore we split the task into two sub-tasks. First we populated the FAQ database using any information sources available. Then, after the system was put into operation, we analyzed the log files.

From the web server logs we could find the most visited information and product pages. Assuming that users may ask about the content of these pages, we created our first FAQs linked to that information. It proved later that the content of the most visited pages did not dominate the information needs posed to the FAQ answering system.

The customer service, whose work the system was supposed to relieve, supplied its own list of FAQs. Some of these FAQs did indeed represent popular information needs, some didn't.

After the FAQ answering system had been in operation for a while, we checked the source of the answers. $65 \%$ of all answers we written specifically for our FAQ database. Only $35 \%$ of the answers were readily available on the company's website. This illustrates the awareness of the company about its customers' information needs. The FAQ answering system improved this awareness considerably.

\section{B. Neighborhood}

The largest restriction of our QA approach is the static nature of FAQs - the answers do not react to details in user queries. This limitation is lessened if the generic answer describes the way to a user-tailored answer. For example, the user may need to log into the self-service system and look for the exact answer there.

It is, in fact, possible to make FAQs more querytailored by linking them to database data [23]. Still, this poses integration difficulties between a QA system and the database. There is no universal solution of how to connect a system to an arbitrary database in a manner similar to how search engines index the web. "'Integration' is a word we don't want to hear" was the message from the company using our system. Even more, the company requested that our software was kept outside its firewalls. The system is an outsourced isolated module that operates isolated FAQs and smoothly fits into the self-service website by means of HTML/HTTP. We do question answering in a plug-n-play manner; no integration with other systems.

There were three considerations that made the company cautious. The spirit of self-service implies reducing the work load on employees; the company was not willing to increase this work load and costs by any kind of system integration. Furthermore, any new third party software inside the firewalls is a security threat. Finally, system integration would probably make the project drown in the internal bureaucracy.

\section{Stable FAQ database}

The initial period, a couple of months, of the system's operation required regular log-mining in order to discover users' information needs and unusual wording of the queries. Log analysis allowed us improving the selection of relevant FAQs and linguistic coverage of question templates.

Only a few new FAQs were added to the database after the initial period of active discovery of the users' information needs, mostly FAQs related to advertizing campaigns and rare external events. The overall subject distribution of user queries is stable. Table 2 shows the share of answered questions in March and October of two consecutive years. The number of submitted questions slowly rises, but the share of answered ones remains stable.

TABLE 2: SHARE OF ANSWERED QUESTIONS OVER TIME.

\begin{tabular}{l|llll}
\hline & Mar & Oct & Mar & Oct \\
\hline Q subm. & 7069 & 9253 & 9341 & 10536 \\
Q answ. & 4974 & 6488 & 6634 & 7450 \\
\% answ. & 70.36 & 70.12 & 71.02 & 70.71 \\
\hline
\end{tabular}

Adding new FAQs and question templates to the database does not cause difficulties because of the granular representation of onto-linguistic knowledge. We should be cautious, however, modifying the shared pieces of onto-linguistic knowledge used by several templates. 


\section{ADVANTAGES OF FAQ ANSWERING FOR WEB SELF-SERVICE}

\section{A. "Intelligent search"}

Let us see how our FAQ answering system overcomes the shortcomings of site search listed in Section II.

1. The FAQ database is populated according to users' information needs being discovered through analysis of user queries. The system pushes forward repeatedly requested answers, which satisfies more than two thirds of all queries.

2. Any QA system is linguistically enhanced. Question templates easily handle synonyms and wording of ontological relationships.

3. The system provides specific answers, documents are included where needed.

4. The system handles all reoccurring queries and delivers definite answers even in cases where site search would yield " 0 documents found".

5. The answers may contain as much multimedia as the medium holding them, in our case HTML and web browsers, allows.

6. Question templates are created by learning from past queries, but they are optimized for future queries disregarding any statistical properties of the text on the website.

7. The FAQ module interprets short queries as questions and answers them accordingly.

\section{B. Business benefits}

The business benefits of automated FAQ answering are twofold. The company that runs the FAQ answering service has acknowledged that its customer service does not receive trivial questions anymore and that operators have more time to deal with complex issues that do require human involvement. A minimized number of repeating insignificant questions to the operators reduces stress and stress-related decrease of productivity. Furthermore, analysis of user queries is a source of business intelligence which helps to monitor customer interests, proactively react to demand, receive feedback on advertizing campaigns. The estimated return on investment was fourfive-fold.

From the user's point of view, automated FAQ answering yields the following benefits: (i) immediate answer, if any, no telephone queues or waiting for an email answer, (ii) availability around the clock, no office hours, (iii) equal availability from around the globe, (iv) interaction in the user's own pace, no one is waiting in the other end of the phone line, (v) comfort for shy people and people with language difficulties.

\section{FURTHER RESEARCH}

There are several angles of the further research. One such angle is that of organizations that employ QA systems. How QA influences knowledge acquisition, storage, retrieval and sharing in an organization? How QA influences productivity, user experience, what is gained and what is lost? A particular case is interaction between users, a self-service system, a QA system, and a customer service.

On the technical side, moving from single-sentence QA to answering multi-line messages, such as e-mail, is a valuable direction of the development. To our knowledge, little research has been done in this direction. The main reason why QA has made so little progress in e-mail answering might be the huge amount of noise in e-mails as queries. Fortunately, our FAQ-based QA has proved successful also with e-mail. Still, there is plenty of space for research.

This paper did not discuss the development and maintenance of the knowledge base. Analysis of past queries, discovering lexical gaps, predicting future queries and their wording is of paramount importance for successful FAQ retrieval. Tool support for development and maintenance of the knowledge base is essential. This includes text clustering, text mining, as well as visualization of semantic proximity between pieces of text.

\section{CONCLUSIONS}

The purpose of this paper is to round up the development of an FAQ answering system and discuss it in the context of web self service where the main task of the system is to outperform its main competitors - site search engines - by providing specific answers and considerably less irrelevant information.

People ask the same questions over and over again; more than two thirds of all questions have proved frequent enough to consider covering them by FAQs. Our system answers correctly more than nine out of ten questions, if the answer is available in the system's database.

The main reason of such good performance is questionspecific representation of onto-linguistic knowledge embodied in a number of question templates linked to the FAQs. The narrow domain is helpful for word sense disambiguation, which includes also multi-word expressions, correct assumptions about words missing in the query, resolving context-dependent synonymy and ontological relations.

We argue that template-based FAQ answering is the most appropriate approach for restricted knowledge domains with up to few hundred FAQs. Being optimized for expected queries, the system demonstrates superior performance and requires a limited knowledge base.

The business benefits of automated FAQ answering are twofold. As website visitors find answers on their own, customer service operators receive fewer trivial questions and have more time for complex issues. Reduced number and increased quality of the questions lessens stress and increases productivity. Analysis of user queries helps to monitor user interests and shape the business according to the demand. From the user's perspective, the system offers instant answers, if any, around the clock from all over the world, allowing inquiries in the user's own pace. 


\section{REFERENCES}

All links were valid in January 2009.

[1] V. Stojanovski, "Web Self-Service Done Right", http://it.toolbox.com/blogs/crm-realms/web-selfservice-done-right8723

[2] "Web Self-Service Lowers Call Center Costs and Improves Customer Service", White Paper, http://www.idsgrp.com/news/ articles/art_bitgroup_cc.pdf

[3] G. Galat, "The Pursuit of Measurement: The Customer's Experience", http://www.webanalyticsassociation.org/en/art/?493

[4] "Google Enterprise: Value of Site Search", http://www.google.com/enterprise/pdf/google_value_sitesearch.pdf

[5] R. Domenig, K. R. Dittrich, "Query Explorativeness for Integrated Search in Heterogeneous Data Sources", Advanced Information Systems Engineering. Springer LNCS, Vol. 2348/2002, ISBN 9783-540-43738-3, 2002, pp. 715-718

[6] M. Chau, F. Xiao, O. R. Liu Sheng, "Analysis of the query logs of a Web site search engine", Journal of the American Society for Information Science and Technology, Vol. 56, Issue 13, 2005, pp. $1363-1376$

[7] W. B. Croft, R. Cook, D. Wilder, "Providing Government Information on the Internet: Experiences with THOMAS", in Proc. Digital Libraries Conference DL '95, 1995, pp. 19-24

[8] J. Lin, "The Web as a Resource for Question Answering Perspective and Challenges", in Proc. LREC, 2002

[9] A. Andrenucci, E. Sneiders, "Automated Question Answering: Review of the Main Approaches", in Proc. 3rd International Conference on Information Technology and Applications (ICITA'05), Sydney, Australia, IEEE Vol. 2, (2005, pp. 514-519

[10] P. Wang, M. W. Berry, Y. Yang, "Mining longitudinal web queries: Trends and patterns", Journal of the American Society for Information Science and Technology, Vol. 54, Issue 8, 2003, pp. 743-758

[11] V. Jijkoun, M. de Rijke, "Retrieving Answers from Frequently Asked Questions Pages on the Web", in Proc. 14th ACM international conference on Information and knowledge management, Bremen, Germany, 2005, pp. 76-83

[12] H. Kim, J. Seo, "High-performance FAQ retrieval using an automatic clustering method of query $\operatorname{logs}$ ", Information Processing and Management: an International Journal, Vol. 42, Issue 3, May 2006, pp. 650-661
[13] J. Jeon, W. B. Croft, J. H. Lee, "Finding similar questions in large question and answer archives", in Proc. 14th ACM international conference on Information and knowledge management, Bremen, Germany, 2005, pp. 84-90

[14] R. Soricut, E. Brill, "Automatic question answering: Beyond the factoid", in Proc. HLT-NAACL, 2004

[15] R. Burke, K. Hammond, V. Kulyukin, S. Lytinen, N. Tomuro, S. Schoenberg, "Question Answering from Frequently-Asked Question Files: Experiences with the FAQ Finder System", AI Magazine, 18(2), 1997, pp. 57-66

[16] W. Winiwarter, "An AdaptiveNatural Language Interface Architecture to Access FAQ Knowledge Bases", in Proc. 4th International Conference on Applications of Natural Language to Information Systems, Vienna, OCG Lecture Notes, Vol. 129, 1999

[17] S. Y. Yang, F. C. Chuang, C. S. Ho, "Ontology-supported FAQ processing and ranking techniques", Journal of Intelligent Information Systems, Vol. 28, Issue 3, June 2007, pp. 233-251

[18] F. Wang, G. Teng, L. Ren, J. Ma, "Research on Mechanism of Agricultural FAQ Retrieval Based on Ontology", in Proc. 2008 Ninth ACIS International Conference on Software Engineering, Artificial Intelligence, Networking, and Parallel/Distributed Computing, 2008, IEEE 2008, pp. 955-958

[19] N. Casellas, P. Casanovas, J. J. Vallbé, M. Poblet, M. Blázquez, J. Contreras, J. M. López-Cobo, V. R. Benjamins, "Semantic enhancement for legal information retrieval: Iuriservice performance", in Proc. 11th international conference on Artificial Intelligence and law, Stanford, California, 2007, pp. 49-57

[20] B. Katz, "Annotating the World Wide Web using natural language", in Proc. RIAO 97, MCGill University, Canada, 1997

[21] E. Sneiders, "Automated FAQ Answering: Continued Experience with Shallow Language Understanding", in Question Answering Systems, Papers from the 1999 AAAI Fall Symposium, November 5 7, North Falmouth, Massachusetts, USA, AAAI Press, pp.97-107

[22] M. Light, G. S. Mann, E. Riloff, E. Breck, "Analyses for elucidating current question answering technology", Journal of Natural Language Engineering, Special Issue on Question Answering, FallWinter, 2001

[23] E. Sneiders, "Automated Question Answering Using Question Templates that Cover the Conceptual Model of the Database", in Proc. Natural Language Processing and Information Systems, NLDB'2002, Stockholm, Sweden, Springer LNCS series 2553, 2002, pp. 235-239 